\title{
Energy Expenditure in Males with Mental Retardation
}

\author{
Hiroko OHWADA ${ }^{1}$, Takeo NAKAYAMA ${ }^{2}$, Yoji SUZUKI ${ }^{3, *}$, Tetsuji YoKoyama ${ }^{4}$ and \\ Masahiko ISHIMARU ${ }^{5}$ \\ ${ }^{1}$ Department of Food Sciences, Ibaraki Christian University, Ibaraki 319-1295, Japan \\ ${ }^{2}$ Department of Health Informatics, Kyoto University School of Public Health, Kyoto 606-8501, Japan \\ ${ }^{3}$ Department of Human Welfare, Sei Gakuin University, Ageo, Saitama 362-0053, Japan \\ ${ }^{4}$ Department of Technology Assessment and Biostatistics, National Institute of Public Health, Wako, Saitama \\ 351-0197, Japan \\ ${ }^{5}$ Department of Health Psychology, Faculty of Literature, Obirin University, Machida, Tokyo 194-0294, \\ Japan
}

(Received June 10, 2004)

\begin{abstract}
Summary Previously, we reported that males with mental retardation (MR) (MR group) expended more energy than males without MR (control group) at a given work load. The precise physiological mechanisms for this difference remain unclear. Using the same set of subjects (23 age-, height-, and weight-matched male pairs, mean age: $36.3 \mathrm{y}$ ), we examined possible causes for the observed metabolic difference by monitoring physical movements and evaluating the metabolic capability of the skeletal muscles. In the supine position when no body movements were detected for any subjects, oxygen consumption $\left(\mathrm{VO}_{2}\right)$ and heart rate (HR) were not markedly different between the MR and the control groups. By contrast, in the sitting and standing positions and during walking at 30 and $50 \mathrm{~m} / \mathrm{min}$, when significantly larger body movements were monitored in the $\mathrm{MR}$ group, $\mathrm{VO}_{2}$ and $\mathrm{HR}$ were significantly higher in this group than in the control group. Linear regression analyses, performed separately in the MR and control groups, revealed that the slope of the regression line of $\mathrm{HR}$ on relative exercise intensity $\left(\% \mathrm{VO}_{2} \max \right)$ during walking, that of $\mathrm{VO}_{2}$ on walking speeds, and that of $\mathrm{VO}_{2}$ on the number of steps in the MR group were almost identical with those in the control group. These results suggest that the capability of skeletal muscles was not so different between the two groups. Thus, the high energy expenditure in the MR group was suggested to be a result of excessive body movements rather than an intrinsic incapability of skeletal muscles.
\end{abstract}

Key Words energy expenditure, oxygen consumption, mental retardation, physical activity, 3-dimensional accelerometry

Fernhall et al. (1) reported that aerobic force, one of the best indices of physical fitness, is $20-40 \%$ lower in individuals with mental retardation (MR) than in those without MR. This difference is thought to be due to the physically inactive lifestyles of people with $\mathrm{MR}(2,3)$. Physical inactivity increases the risk of cardiovascular disease (CVD) (4), which is more prevalent among people with MR than among people without MR $(5,6)$. Therefore, in order to prevent CVD in people with MR, it may be important to modify their lifestyles. Few studies have examined the characteristics of energy expenditure during activities of daily life (ADL) among people with MR, which is essential in planning a physical exercise program.

Previously, we reported that males with MR expended more energy than males without MR (7). We suggested that either extra movements, such as bodyswinging, or an intrinsic metabolic abnormality may result in a difference in the energy expenditure between

\footnotetext{
${ }^{*}$ He died in September, 2003.

E-mail: h.ohwada@icc.ac.jp
}

people with and without MR.

To explore the possible mechanisms of the observed metabolic difference, we used the same subjects as in our previous study, but examined both the individual's physical movements and the metabolic capability of the skeletal muscles. We combine the results of this study with our previous findings and present a comprehensive discussion.

\section{METHODS}

Subjects. The subjects were 23 males with MR (aged 18- 49 y) (MR group) that live in either of two special facilities in Ibaraki Prefecture, Japan. The IQ level (mean \pm SD: $35.5 \pm 10.3$, and range: $21-59$ ) was determined according to the Tanaka-Binet method (8). None of the subjects suffered from a manifest physical handicap (e.g., cerebral palsy), Down's syndrome, arrhythmia, hypertension, ischemic heart disease, or other heart diseases. In addition, none of the subjects were under any medication known to affect energy expenditure (e.g., steroids or thyroxine).

The control subjects were 23 non-athlete male vol- 
unteers without MR (aged 20-48 y) (control group), who were individually matched to the MR group for age ( $\pm 3 \mathrm{y})$, body height $( \pm 4 \mathrm{~cm})$, and weight $( \pm 9 \mathrm{~kg})$. For matching criteria, one standard deviation of each variable was used. Table 1 shows the characteristics of both the MR group and the control group.

The study protocol was approved by the Ethical Review Committee of the Medical Research Institute, Tokyo Medical and Dental University. Informed consent was obtained from each subject or his legal guardian.

Experimental procedure. The examination was conducted at both of the residential facilities. The details were reported previously (7). Briefly, individuals of the control group reported to their assigned facility and stayed overnight. Each of the subjects had supper before $1900 \mathrm{~h}$ and went to bed before 2200. Basal metabolic rate (BMR) was measured between 0530 and 0700 the next morning. The procedures were conducted according to the standardized protocols that include starting the procedure after an overnight fast $\geq 10 \mathrm{~h}$, remaining in bed prior to awakening, no physiological or psychological stress, and a thermoneutral $\left(25^{\circ} \mathrm{C}\right)$, semi-darkened environment. After determining the BMR, the subjects were asked to void. They then underwent anthropometric measurements. These procedures were followed by a light breakfast and a $2 \mathrm{~h}$ rest period. At the end of the rest period, chest electrodes and a 3dimensional accelerometer were attached to the subjects to measure and record heart rate (HR) and body movement. The subjects were placed in a supine position on a comfortable mat for $30 \mathrm{~min}$, after which data collection was started. Oxygen consumption $\left(\mathrm{VO}_{2}\right), \mathrm{HR}$, and body movement were measured while the subjects were lying in a supine position, sitting comfortably in a chair, standing upright, walking, or pedaling a bicycle ergometer. HR was measured and recorded throughout the examination using a telemeter (three chest leads; Biomulti1000, PN1721, NEC Medical Systems Co., Tokyo, Japan) under the supervision of a physician.

Anthropometry and body composition. Height and body mass were measured in accordance with standard procedures (9). Body composition was assessed by densitometry with a $\mathrm{SF}_{6}$ dilution technique (BSF-200, Shimadzu Corporation, Kyoto, Japan) $(10,11)$. The body volume $V$ was computed as follows:

$$
V=V_{0}+V_{1}-\left(V_{1} \times 10^{6}\right) / X \text {, }
$$

where $\mathrm{V}_{0}$, the volume of the chamber $(195 \mathrm{~L}) ; V_{1}$, the amount of diffused $\mathrm{SF}_{6}$ after attaining equilibrium; $X$, the concentration of $\mathrm{SF}_{6}$ gas (ppm). The body density (body mass/body volume), body fat (\%) (12), and lean body mass (LBM) (body mass - fat mass) were calculated.

Measurement of basal, resting, and walking metabolisms. Basal, resting, and walking metabolisms were measured by indirect calorimetry using the Douglas bag technique (13). The method of BMR measurement was based on the DuBois (14) procedure. The BMR was measured by collecting two consecutive 7 min samples of expired air. Resting metabolism was measured by collecting the expired air of the subject for $10 \mathrm{~min}$ while he was in the supine position, for $5 \mathrm{~min}$ in the sitting position, and for $5 \mathrm{~min}$ in the standing position. Walking metabolism was then measured as the subjects walked around a track (circumference: $60 \mathrm{~m}$ ) at speeds of 30 , 50 , and $70 \mathrm{~m} / \mathrm{min}$. The track was equipped with timing lights at 2-m intervals which lit in sequence and paced the subjects at a standardized walking speed (Paceleader, PL-100C, Yagami Inc., Nagoya, Japan). The total elapsed time of the trial was $12 \mathrm{~min}$. The expired air was collected using the Douglas bag for the last $2 \mathrm{~min}$ for each walking speed.

Estimation of maximal oxygen consumption. A maximal exercise test was performed using a progressive submaximal workload protocol. A computer-controlled bicycle ergometer (FFS, Bridgestone Cycle Co., Tokyo, Japan) was used. Each subject exercised at three workloads that corresponded to 40,50 , and $60 \%$ of his own maximal oxygen consumption $\left(\mathrm{VO}_{2} \mathrm{max}\right)$, which was estimated for his age. Each load was maintained for $4 \mathrm{~min}$, and the three loads were applied consecutively

Table 1. Characteristics of the subjects.

\begin{tabular}{lcrc}
\hline \multicolumn{1}{c}{ Variable } & $\begin{array}{c}\text { Subjects with mental } \\
\text { retardation(MR group) } \\
(n=23)\end{array}$ & $\begin{array}{c}\text { Subjects without mental } \\
\text { retardation (Control group) } \\
(n=23)\end{array}$ & $p^{\mathrm{a}}$ \\
\hline Age $(\mathrm{y})$ & $36.3(8.9)$ & $36.3(8.0)$ & 1.00 \\
Height $(\mathrm{cm})$ & $164.2(4.1)$ & $164.4(3.7)$ & 0.65 \\
Weight $(\mathrm{kg})$ & $62.4(12.6)$ & $63.6(6.9)$ & 0.68 \\
$\mathrm{BMI}\left(\mathrm{kg} / \mathrm{m}^{2}\right)$ & $23.1(4.1)$ & $23.5(2.5)$ & 0.66 \\
$\mathrm{LBM}(\mathrm{kg})^{\mathrm{b}}$ & $50.7(8.2)$ & $52.2(5.7)$ & 0.47 \\
$\mathrm{Body} \mathrm{fat}(\%)^{\mathrm{b}}$ & $19.7(6.3)$ & $17.1(5.8)$ & 0.19 \\
$\mathrm{VO}{ }_{2} \mathrm{max}$ & & $41.3(10.0)$ & 0.039 \\
$(\mathrm{~mL} / \mathrm{kg} / \mathrm{min})$ & $34.0(9.0)$ & $50.7(9.5)$ & 0.038 \\
$(\mathrm{~mL} / \mathrm{kg} \mathrm{LBM} / \mathrm{min})$ & $41.8(11.1)$ & \\
\hline
\end{tabular}

Values are given as the mean (SD).

${ }^{a} p$, MR group vs control group by paired $t$-test.

${ }^{\mathrm{b}}$ Values determined by the $\mathrm{SF}_{6}$ dilution method.

BMI: body mass index, LBM: lean body mass, $\mathrm{VO}_{2}$ max: maximal oxygen consumption. 
Table 2. Oxygen consumption, heart rate, and body movement during measurement of basal metabolic rate (BMR) and resting metabolic rate in the different positions in subjects of the MR group and the control group.

\begin{tabular}{|c|c|c|c|}
\hline Variable & MR group $(n=23)$ & Control group $(n=23)$ & $p$ \\
\hline \multicolumn{4}{|l|}{ Basal metabolic rate (BMR) } \\
\hline Oxygen consumption (mL/kg/min) & $3.30(0.56)$ & $3.19(0.41)$ & $0.509^{\mathrm{a}}$ \\
\hline Heart rate (beats/min) & $60.0(6.2)$ & $60.5(6.3)$ & $0.817^{\mathrm{a}}$ \\
\hline \multicolumn{4}{|l|}{ Resting metabolic rate } \\
\hline \multicolumn{4}{|l|}{ Supine position } \\
\hline Oxygen consumption $(\mathrm{mL} / \mathrm{kg} / \mathrm{min})$ & $3.76(0.55)$ & $3.52(0.42)$ & $0.18^{\mathrm{a}}$ \\
\hline Heart rate (beats/min) & $63.8(10.7)$ & $58.0(8.1)$ & $0.06^{\mathrm{a}}$ \\
\hline Body movement $\left(\mathrm{m} / \mathrm{s}^{2}\right)^{\mathrm{c}}$ & $0.000(0.000)$ & $0.000(0.000)$ & - \\
\hline \multicolumn{4}{|l|}{ Sitting position } \\
\hline Oxygen consumption $(\mathrm{mL} / \mathrm{kg} / \mathrm{min})$ & $4.33(0.66)$ & $3.73(0.46)$ & $0.002^{\mathrm{a}}$ \\
\hline Heart rate (beats/min) & $71.4(9.1)$ & $61.8(8.8)$ & $0.001^{\mathrm{a}}$ \\
\hline Body movement $\left(\mathrm{m} / \mathrm{s}^{2}\right)^{\mathrm{c}}$ & $0.037(0.049)$ & $0.000(0.000)$ & $<0.001^{b}$ \\
\hline \multicolumn{4}{|l|}{ Standing position } \\
\hline Oxygen consumption $(\mathrm{mL} / \mathrm{kg} / \mathrm{min})$ & $4.61(0.87)$ & $3.95(0.63)$ & $0.003^{\mathrm{a}}$ \\
\hline Heart rate (beats/min) & $78.9(11.0)$ & $68.2(9.8)$ & $0.001^{\mathrm{a}}$ \\
\hline Body movement $\left(\mathrm{m} / \mathrm{s}^{2}\right)^{\mathrm{c}}$ & $0.064(0.048)$ & $0.000(0.000)$ & $<0.001^{b}$ \\
\hline
\end{tabular}

Values are given as the mean (SD).

${ }^{a} p$, MR group vs. control group by paired $t$-test.

${ }^{b}$ Fisher's exact test (2-tail) where acceleration is classified as zero (below the sensitivity of the accelerometer) or nonzero.

${ }^{\mathrm{c}}$ The average magnitude (above $0.002 \mathrm{G}$ ) of a composite of the 3-dimensional acceleration vectors.
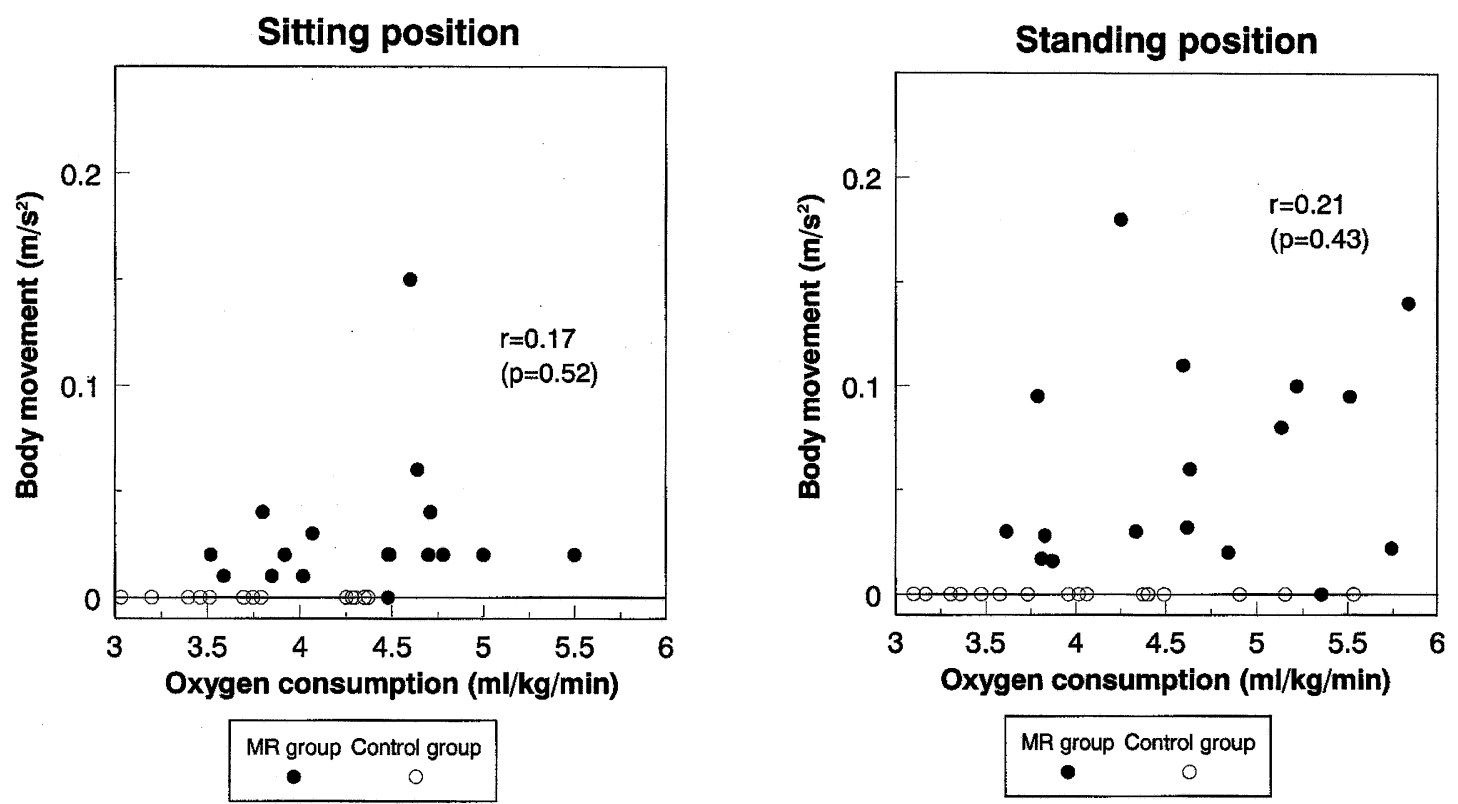

Fig 1. Relationship between oxygen consumption and body movement in sitting and standing positions in subjects with mental retardation (MR group, closed circle) and without mental retardation (control group, open circle).

for a total of $12 \mathrm{~min}$. Expired air was collected in the Douglas bag for the last $1 \mathrm{~min}$ of each load. After a graph was prepared for $\mathrm{HR}$ versus $\mathrm{VO}_{2}$ at the three load levels, a regression line was drawn. With the assumption that the maximal HR (HRmax, beats/min) can be calculated as 220 minus the subject's age in years, the $\mathrm{VO}_{2}$ max was estimated as the $\mathrm{VO}_{2}$ at HRmax.

Measurement of oxygen consumption. The oxygen and carbon dioxide content of the expired air were measured with an expired air analyzer (Expired Gas Monitor 1H26, NEC Medical Systems, Co.). The total volume of expired air was measured with a dry gas meter (DC-5A, Shinagawa Factory, Tokyo, Japan) and was corrected for temperature, pressure, and humidity.

Measurement of body movement with an accelerometer. Body movement was measured using a 3-dimensional accelerometer (Activetracer, AC-300, GMS Co., Tokyo, Japan), which records the magnitudes (above $0.002 \mathrm{G}$ ) of the 3-dimensional acceleration vectors and their composition every $40 \mathrm{~ms}$. In this study, this device was set to yield the average composite acceleration (above $0.002 \mathrm{G}$ ) of the magnitudes of the 3-dimensional accel- 
Table 3. Oxygen consumption, heart rate, body movement, and number of steps during the different speeds of walking in subjects of the MR group and the control group.

\begin{tabular}{|c|c|c|c|}
\hline Variable & MR group $(n=23)$ & Control group $(n=23)$ & $p$ \\
\hline \multicolumn{4}{|l|}{ Walking speed $(30 \mathrm{~m} / \mathrm{min})$} \\
\hline Oxygen consumption $(\mathrm{mL} / \mathrm{kg} / \mathrm{min})$ & $9.27(1.44)$ & $8.35(0.96)$ & $0.025^{\mathrm{a}}$ \\
\hline Heart rate (beats/min) & $85.8(14.4)$ & $74.5(10.1)$ & $0.007^{\mathrm{a}}$ \\
\hline Body movement $\left(\mathrm{m} / \mathrm{s}^{2}\right)^{\mathrm{c}}$ & $1.41(0.21)$ & $0.95(0.18)$ & $<0.001^{\mathrm{b}}$ \\
\hline Number of steps/min & $86.1(11.6)$ & $76.0(10.2)$ & $0.001^{\mathrm{a}}$ \\
\hline \multicolumn{4}{|l|}{ Walking speed (50 m/min) } \\
\hline Oxygen consumption (mL/kg/min) & $11.33(2.15)$ & $10.08(1.54)$ & $0.033^{\mathrm{a}}$ \\
\hline Heart rate (beats/min) & $93.0(15.6)$ & $79.6(10.3)$ & $0.004^{\mathrm{a}}$ \\
\hline Body movement $\left(\mathrm{m} / \mathrm{s}^{2}\right)^{\mathrm{c}}$ & $2.42(0.44)$ & $1.72(0.34)$ & $<0.001^{b}$ \\
\hline Number of steps/min & $106.3(10.3)$ & $98.5(8.1)$ & $0.005^{\mathrm{a}}$ \\
\hline \multicolumn{4}{|l|}{ Walking speed $(70 \mathrm{~m} / \mathrm{min})^{\mathrm{d}}$} \\
\hline Oxygen consumption (mL/kg/min) & $13.40(2.35)$ & $13.02(2.13)$ & $0.53^{\mathrm{a}}$ \\
\hline Heart rate (beats/min) & $101.6(19.1)$ & $86.8(9.0)$ & $0.003^{\mathrm{a}}$ \\
\hline Body movement $\left(\mathrm{m} / \mathrm{s}^{2}\right)^{\mathrm{c}}$ & $3.46(0.77)$ & $2.60(0.58)$ & $<0.001^{b}$ \\
\hline Number of steps/min & $120.2(9.2)$ & $112.0(10.1)$ & $0.003^{\mathrm{a}}$ \\
\hline
\end{tabular}

Values are given as the mean (SD).

${ }^{a} p$, MR group vs. control group by paired $t$-test.

${ }^{\mathrm{b}} p$, MR group vs. control group by unpaired $t$-test.

${ }^{\mathrm{c}}$ The average magnitude (above $0.002 \mathrm{G}$ ) of a composite of the 3-dimensional acceleration vectors.

d 17 of 23 subjects with MR could not keep up with the speed of $70 \mathrm{~m} / \mathrm{min}$ (see "Results").
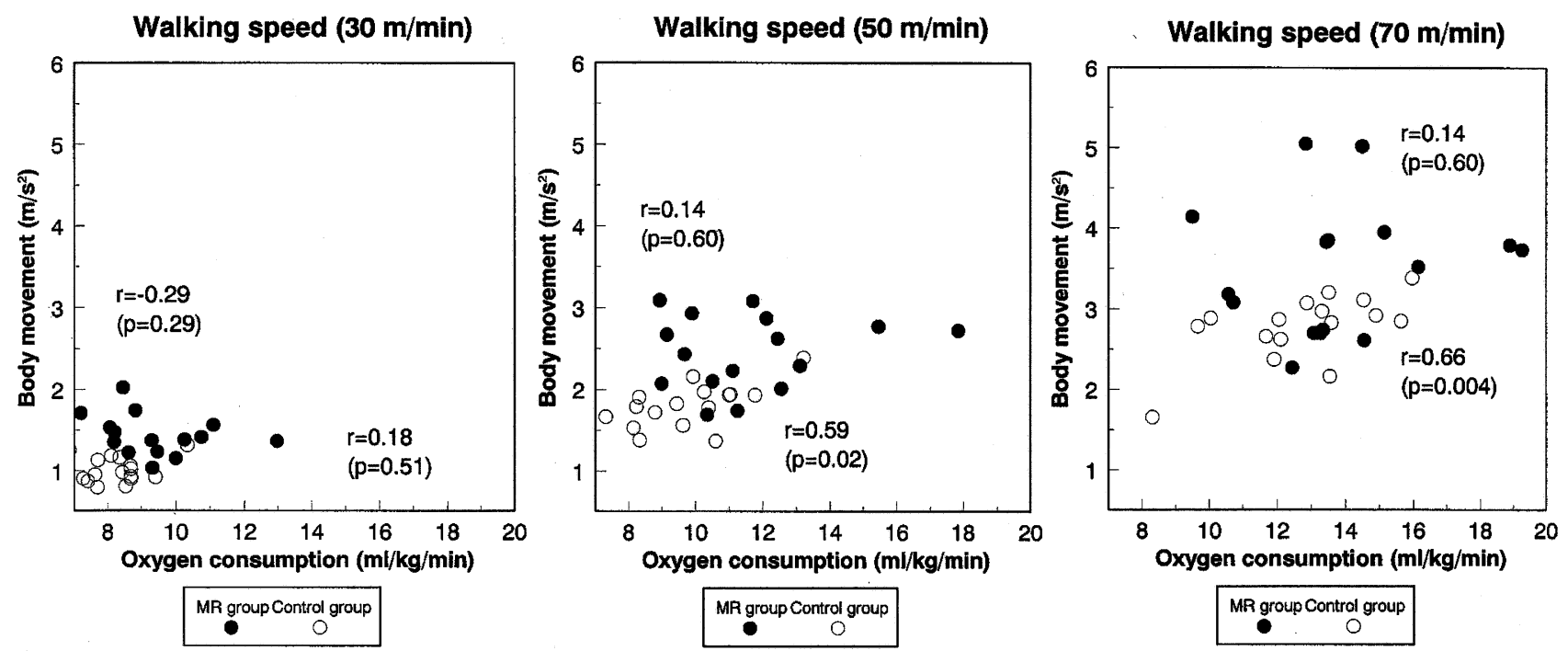

Fig 2. Relationship between oxygen consumption and body movement during 30,50, and $70 \mathrm{~m} / \mathrm{min}$ walking speeds in subjects with mental retardation (MR group, closed circle) and without mental retardation (control group, open circle).

eration vector every $20 \mathrm{~s}$.

Statistical analysis. Differences between the data for the MR group and the control group were assessed using a paired $t$-test or $t$-test. Values for a two-sided test $(p<0.05)$ were considered statistically significant. Correlation and linear regression analyses were done separately for the MR group and for the control group to assess the relationships between walking speed and $\mathrm{VO}_{2}$, between the number of steps and $\mathrm{VO}_{2}$, and between relative exercise intensity $\left(\% \mathrm{VO}_{2}\right.$ max $)$ and $\mathrm{HR}$ during walking. All analyses were conducted with a SPSS ver. 10.0 (SPSS Inc.).

\section{RESULTS}

The $\mathrm{VO}_{2} \max (\mathrm{mL} / \mathrm{kg} / \mathrm{min})$ for the MR group was $18.0 \%$ less than that of the control group ( $p=0.039)$. In addition, the $\mathrm{VO}_{2} \max (\mathrm{mL} / \mathrm{kg} \mathrm{LBM} / \mathrm{min})$ was lower in the MR group by $17.8 \%(p=0.038)$ (Table 1$)$.

In the basal state and supine position, no marked differences were detected between the two groups with respect to $\mathrm{VO}_{2}$ and $\mathrm{HR}$ (Table 2). However, $\mathrm{VO}_{2}$ and $\mathrm{HR}$ in the sitting position and those in the standing position were higher for the MR group by $17.7 \%(p=0.002)$, $17.3 \%(p=0.001), 18.6 \% \quad(p=0.003)$, and $17.6 \%$ 


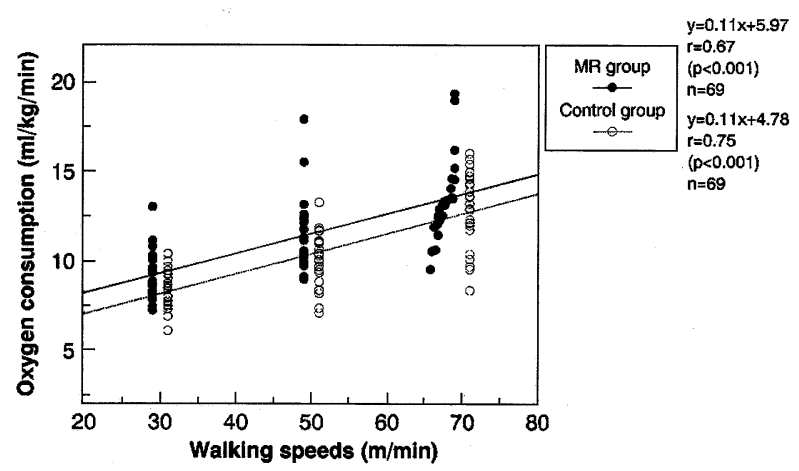

Fig 3. Relationship between walking speeds and oxygen consumption in subjects with mental retardation (MR group, closed circle) and without mental retardation (control group, open circle).

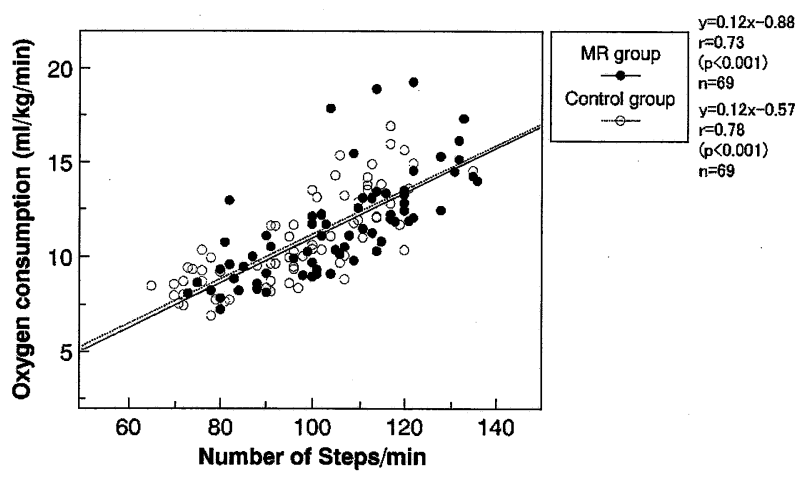

Fig 4. Relationship between number of steps and oxygen consumption in subjects with mental retardation (MR group, closed circle) and without mental retardation (control group, open circle).

( $p=0.001)$, respectively. Vacillation of the center of gravity was measured in 17 individuals of the MR group and 16 individuals of the control group. No body movement was detected for any of the 33 subjects in a supine position. However, subtle body movements were recorded in 16 of the 17 subjects with MR in the sitting and standing positions, while no body movements were detected in the control subjects (Fig. 1).

Table 3 and Fig. 2 present the $\mathrm{VO}_{2}$, HR, body movements, and number of steps taken per min (Walking Steps Rate, WSR) during walking. For both groups, all variables were rectilinearly correlated to walking speed. At $30 \mathrm{~m} / \mathrm{min}$ speed, $\mathrm{VO}_{2}, \mathrm{HR}$, body movements, and WSR for the MR group were higher than those of the control group [by $12.5 \%$ ( $p=0.025), 17.7 \%$ ( $p=0.007)$, $59.9 \%(p<0.001)$, and $14.7 \%(p=0.001)$, respectively]. At $50 \mathrm{~m} / \mathrm{min}$ speed, $\mathrm{VO}_{2}, \mathrm{HR}$, body movements, and WSR were also higher than those of the control group [by $14.1 \%$ ( $p=0.033), 19.4 \%(p=0.004), 50.4 \%(p<$ $0.001)$, and $8.7 \%(p=0.005)$, respectively]. At the examination for $70 \mathrm{~m} / \mathrm{min}$ speed, 17 of the 23 subjects with MR could not maintain this speed for $4 \mathrm{~min}$, and the mean walking speed of the MR group remained at $65 \mathrm{~m} / \mathrm{min}$. Despite their slower walking speed, the $\mathrm{VO}_{2}$, HR, body movements, and WSR were higher than those of the control group [by 5.4\% (not significant), 18.4\%

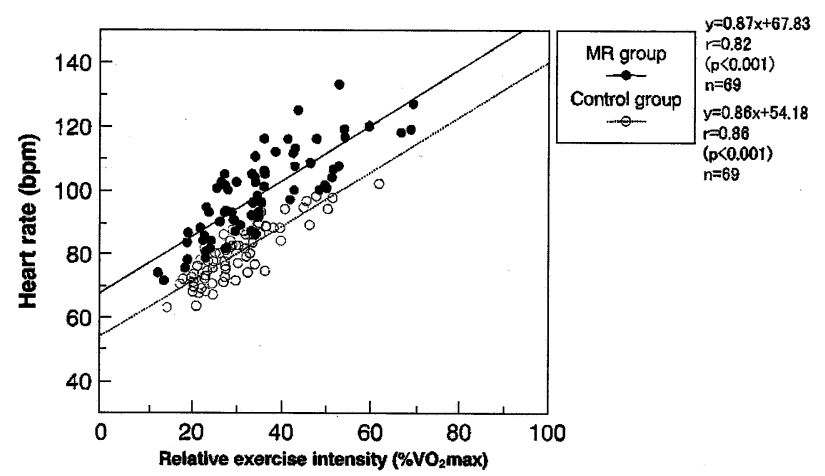

Fig 5. Relationship between relative exercise intensity $\left(\% \mathrm{VO}_{2} \max \right)$ and heart rate during walking in subjects with mental retardation (MR group, closed circle) and without mental retardation (control group, open circle).

$(p=0.003), 51.2 \%(p<0.001)$, and $8.0 \%(p=0.003)$, respectively].

The relationship between walking speed and $\mathrm{VO}_{2}$ is demonstrated in Fig. 3. There were significant correlations in both groups. Interestingly, the regression line for the MR group was located at a higher position than that for the control group, and the two lines were approximately parallel, which meant that the $\mathrm{VO}_{2}$ was systematically higher in the MR group than that in the control group when the walking speeds of both groups were same.

Figure 4 demonstrates the relationship between the WSR and $\mathrm{VO}_{2}$ regardless of walking speed. A rectilinearly correlated relationship was also uncovered in both the MR and the control groups. The two regression lines were approximately overlapped with each other.

Figure 5 reveals a significant correlation between the $\% \mathrm{VO}_{2}$ max and $\mathrm{HR}$ during walking in both groups. In a similar manner as seen in Fig. 1, the regression lines for the MR and control groups were parallel, indicating that $\mathrm{HR}$ at a given $\% \mathrm{VO}_{2}$ max was systematically higher in the MR group than in the control group.

\section{DISCUSSION}

We previously reported that $\mathrm{VO}_{2}, \mathrm{HR}$, and body movements at a given walking speed were significantly higher in the MR group than those in the control group (7). In the present study, there were no differences between the two groups in WSR or in increments (\%change) of $\mathrm{VO}_{2}$ with an increasing walking speed; the regression line slopes in both groups were almost identical. When slope is constant, energy expenditure for walking can be determined by extent of mobilization in related active muscles per unit time. Therefore, these results suggest that the metabolic capability of skeletal muscle was similar between the two groups.

Although maximal exercise testing is the gold standard for measuring aerobic capacity, this technique is difficult to use in many populations that have disabilities (15). $\mathrm{VO}_{2}$ max was computed by interpolating predicted age-adjusted HRmax in the regression equation 
of $\mathrm{VO}_{2}$ on $\mathrm{HR}$; data were obtained during steady exercise at three different work loads of moderate intensity (16). The formula for estimating $\mathrm{VO}_{2}$ max was prepared for each individual in both the MR and control group. Nonetheless, uncertainty exists as to whether this formula is appropriate for individuals with MR. It is well established that HRmax is reduced with increasing age in individuals without MR, but such a relationship remains unclear in individuals with MR. In the present study, HR for walking is linearly related to $\% \mathrm{VO}_{2} \max$, and no marked differences were found in this relationship between trained and non-trained persons (data not shown). Furthermore, no individual in the MR group suffered from heart disease. These results suggest that HRmax can be predicted for individuals in the MR group by using the same equation that applies to individuals without MR. Thus the estimated $\mathrm{VO}_{2}$ max in the present study can be used to evaluate differences between individuals with and without MR.

The $\mathrm{VO}_{2}$ max or $\mathrm{VO}_{2}$ peak was lower in individuals with MR than in individuals without MR by 20 to $40 \%$ (1), by 30\% (17). In addition, Morgan (18) reported that the predicted $\mathrm{VO}_{2}$ max in depressed psychiatric females was $24 \%$ lower than that in healthy females. The present study shows that $\mathrm{VO}_{2}$ max in the MR group was lower than that in the control group by $18.0 \%$ which is comparable to the previous reports $(1,17,18)$.

A reduced $\mathrm{VO}_{2}$ max was observed in the MR group even though individuals in both the MR and control groups had a similar body composition. It is difficult to discuss the reason for this difference because no direct measurements of $\mathrm{VO}_{2} \max$ (e.g., cardiac output during moderate or maximal exercise) were taken. Given that the degree of physical activity performed during free time strongly affects $\mathrm{VO}_{2} \max$, a decrease in $\mathrm{VO}_{2}$ max may be related to the prolonged sedentary situation in daily free time among individuals with MR (19).

A standing position is maintained by the postural reflex and anti-gravity muscles. However, since persons with psychiatric anomalies often develop a head fluctuation in the standing position, an increase in work is often found in the anti-gravity muscles and cervical/ neck reflex in order to maintain posture while resting. In fact, increased head fluctuation in the MR group, which was not observed in the control, suggests a significant shifting in the body's center of gravity while standing. This unique body swing in MR individuals could result in an increase in muscle fiber of the antigravity muscles, which are necessary to maintain a standing position while at rest. Accordingly, this could explain why $\mathrm{VO}_{2}$ and $\mathrm{HR}$ in the MR group were significantly higher when in the sitting and standing positions.

Typical ADL in individuals with MR consists of mild walking at speeds of 30 and $50 \mathrm{~m} / \mathrm{min}$. When a mild work load was applied at these speeds in both groups with a comparable body mass and LBM, increases in WSR and body swing with head fluctuation were observed in the MR group; this resulted in a $10 \%$ increase in $\mathrm{VO}_{2}$. A previous study that used cycling as a measurement showed that a higher $\mathrm{VO}_{2}$ resulted when leg movement was increased as constant work load (20). This suggests that the required energy includes the standing position $\mathrm{VO}_{2}$ in addition to other work components of walking, e.g., body swing frequency and kinetic work in the movements of the legs. In fact, WSR was rectilinearly correlated to $\mathrm{VO}_{2}$ during standardized walking in both groups.

Heart rate during walking was linearly related to $\mathrm{VO}_{2}$ and $\mathrm{HR}$ response to $\% \mathrm{VO}_{2} \max$ was similar between groups. These observations illustrate that $\mathrm{VO}_{2}$ in the MR group was higher than the control group for standardized ADL while walking and while in an anti-gravity posture. Intensity could also be estimated by calculating HR response.

Sixteen individuals of the MR group were unable to maintain a $70 \mathrm{~m} / \mathrm{min}$ speed while six individuals had no difficulty. The 16 individuals who had difficulty could not maintain approximately 120 WSR while walking at a speed of $70 \mathrm{~m} / \mathrm{min}$; this was close to the upper threshold of their ambulatory motion. The WSR at this speed was similar to a pedaling speed of $60 \mathrm{rpm}$ in which leg movements often become difficult for individuals with MR. Needless to say, this heavy work load is rarely found in the ADL of MD individuals.

$\mathrm{VO}_{2}$ showed a linear relationship when plotted against external work (walking speed) or against WSR and, therefore, can be represented by the equation, $y=a+b x$. The reciprocal of $b$ can be used as an expression for efficiency, called delta efficiency (20). No differences were observed in $b$ between the MR group and the control group, which suggests that delta efficiency is similar in both groups. This further indicates that both groups were similar in ADL with respect to mechanical and metabolic characteristics.

This study has shown that energy expenditure in individuals with MR increases with excess body movement despite healthy metabolic function. Accordingly, these findings suggest that energy indices used for healthy individuals (e.g. the 'Mets') are inapplicable to the assessment of energy expenditure during ADL of MR individuals. In order to determine a suitable energy requirements for people with MR, further studies are needed on energy expenditure.

\section{REFERENCES}

1) Fernhall B, Tymeson GT, Webster GE. 1988. Cardiovascular fitness of mentally retarded individuals. Adapt Phys Activity Q 5: 12-28.

2) Fernhall B, Tymeson G. 1987. Graded exercise testing of mentally retarded adults: a study of feasibility. Arch Phys Med Rehabil 68: 363-365.

3) Pitetti KH, Tan DM. 1990. Cardiorespiratory responses of mentally retarded adults to air brake ergometry and treadmill exercise. Arch Phys Med Pehabil 71: 318-321.

4) Paffenbarger RS Jr, Hyde RT, Wing AL, Hsieh CC. 1986. Physical activity, all-cause mortality, and longevity of college alumni. N Engl J Med 314: 605-613.

5) Janicki MP, Jacobson JW. 1982. The character of developmental disabilities in New York State: preliminary observations. Int J Rehabil Res 5: 191-202. 
6) Janicki MP, MacEachron AE. 1984. Residential, health, and social service needs of elderly developmentally disabled persons. Gerontologist 24: 128-137.

7) Iwaoka H, Yokoyama T, Masayasu S, Fuchi T, Nakayama T, Tanaka H. 1998. Characteristics of energy metabolism in males with mental retardation. J Nutr Sci Vitaminol 44: 151-164.

8) Tanaka Institute for Education. 1987. Tanaka-Binet Intelligence Scale. Taken Publisher Co., Tokyo.

9) Lohman T, Roche KA, Martorell R. 1991. Anthropometric Standardization Reference Manual. Human Kinetics Books, Champaign.

10) Hattori K, Matsuzaka A, Iwaoka H, Ohno K, Mitani H. 1994. Determination of body volume in normal adults and in patients with cerebral palsy by the sulfur hexafluoride $\left(\mathrm{SF}_{6}\right)$ dilution technique. Am J Hum Biol 6: 651-657.

11) Iwaoka H, Yokoyama T, Matsumura Y, Yoshitake Y, Fuchi T, Nakayama T, Yoshiike N, Tanaka H. 1998. Determination of percent body fat by newly developed sulfur hexafluoride dilution method and air displacement plethysmography. J Nutr Sci Vitaminol 44: 561568.

12) Brozek J, Grande F, Anderson JT, Keys A. 1963. Densitometric analysis of body composition: revision of some quantitative assumptions. Ann NY Acad Sci 110: 113140.
13) Douglas CG. 1911. A method for determining the total respiratory exchange in man. J Physiol 42: 17-18.

14) DuBois EF. 1936. Basal Metabolism in Health and Disease, 3rd ed. Lea and Febiger Press, Philadelphia.

15) Fernhall B, Unnithan VB. 2002. Physical activity, metabolic issues, and assessment. Phys Med Rehabil Clin N Am 13: 925-947.

16) Blair SN. 1984. Behavioral Health. In: A Handbook of Health Enhancement and Disease Prevention (Matarazzo JD et al., eds), p 438. John Wiley and Sons, New York.

17) Chaiwanichsiri D, Sanguanrungsirikul S, Suwannakul W. 2000. Poor physical fitness of adolescents with mental retardation at Rajanukul School, Bangkok. J Med Assoc Thai 83: 1387-1392.

18) Morgan WP. 1970. Physical work capacity in depression and non-depression psychiatric females: A preliminary study. Am Corr Ther J 24: 14-16.

19) Saltin B. 1990. Cardiovascular and pulmonary adaptation to physical activity. In: Excise, Fitness, and HealthA Consensus of Current Knowledge (Bouchard C, Shephard RJ, Stephens T, Sutton JR, McPherson BD, eds), Cp.18, p 187-203. Human Kinetics Books, Champaign.

20) Suzuki Y. 1979. Mechanical efficiency of fast- and slowtwitch muscle fibers in man during cycling. J Appl Physiol: Respirat Environ Exercise Physiol 47:263-267. 